\title{
AN EMBEDDING CHARACTERIZATION OF ALMOST COMPACT SPACES
}

\author{
BY \\ SERGIO SALBANY
}

Dedicated to Carlos Vilhena on the occasion of his 90 th birthday

\begin{abstract}
We characterize almost compact and almost realcompact spaces in terms of their situation in the product $(J, u)^{C}$. In the characterization of almost compactness $J$ is the two point set or the unit interval; in the characterization of almost realcompactness $J$ is the set of nonnegative integers or the nonnegative reals. $u$ is the upper topology on the real line restricted to $J$.
\end{abstract}

1. Introduction. Amongst the most beautiful representation theorems for topological spaces are the characterization of compact Hausdorff spaces as closed subspaces of products of copies of the unit interval $[0,1]$ (with its usual topology) and the characterization of realcompact spaces as closed subspaces of products of copies of the real line $\mathbf{R}$ also endowed with its usual topology (see [3]). In [2], Frolik and Liu gave an interesting characterization of almost compact and almost realcompact spaces as maximal $T_{2}$ subspaces of their closure in the canonical product of copies of the nonnegative reals with the upper topology $\Pi\left\{\mathbf{R}_{f}^{+} \mid f \in C_{+}(X)\right\}$.

The present paper tries to clarify the role of the maximal $T_{2}$ condition and the extent to which almost compact and almost realcompact spaces can be regarded as "closed" subspaces of the canonical product of a standard object, but where "closure" is not necessarily the closure in the usual product topology.

There is an interesting variation on this theme which arises from changing the standard object. In this direction Kate Halpin [4] has obtained the same characterizations as Z. Frolik and C. T. Liu, the standard spaces $[0,1]$ and $\mathbf{R}^{+}$being replaced by the two point space $\{0,1\}$ and the nonnegative integers. Throughout this paper we shall present our proofs in a way that unifies these two situations.

In a sense, this paper is a discussion of almost compact and almost realcompact spaces from the viewpoint of [12].

2. The canonical product. Let $J \subset \mathbf{R}$ be either the two point set $D=\{0,1\}$ or the unit interval $I=[0,1]$. There are three topologies on $\mathbf{R}$ and $J$ that are important in our discussion: the upper topology $u$ with basic open sets of the form $(-\infty, x)$; the lower topology $l$ with basic open sets $(x,+\infty)$ and the usual topology $u \vee l$. We shall represent the set of all continuous functions on a topological space $(X, T)$ to

Received by the editors March 13, 1979 and, in revised form, December 3, 1981.

1980 Mathematics Subject Classification. Primary 54C25, 54C99; Secondary 54D30, 54B10.

Key words and phrases. Almost compact, almost realcompact, open ultra-filter, maximal relatively separated subspaces, Fomin extension, embedding, canonical product.

(C) 1983 American Mathematical Society 0002-9947/81/0000)-0307/\$03.75 
$(J, u)$ by $C$ and the corresponding product map from $X$ to $J^{C}$ by $e$. The three topologies on $J$ give rise to three topologies on the product $J^{C}$ which we shall represent as $(J, u)^{C}=\Pi(J, u)_{f}=\left(\Pi J_{f}, \Pi u\right) ;(J, l)^{C}=(\Pi J, \Pi l)$ and $(J, u \vee l)^{C}=$ $(\Pi J, \Pi(u \vee l))=(\Pi J, \Pi u \vee \Pi l)$. We denote by $(J, u, l)$ the bitopological space $J$ equipped with the two topologies $u$ and $l$, and the resulting bitopological product space by $(J, u, l)^{C}=\left(J^{C}, \Pi u, \Pi l\right)$.

We refer to [6,9 and 11] for the theory of bitopological spaces that is needed.

3. Some properties of the embedding $e: X \rightarrow J^{C}$.

Lemma 1. Let $\alpha$ be in the $\Pi(u \vee l)$-closure $e[X]$ in the product $(J, u, l)^{C}$. Let $h:(J, u) \rightarrow(J, u)$. For any $f$ in $C, h \circ f$ is in $C$ and $\alpha_{h \circ f} \leqslant h\left(\alpha_{f}\right)$.

Proof. Suppose there is $\beta$ such that $h\left(\alpha_{f}\right)<\beta<\alpha_{h \circ f}$. By continuity of $h$ and $\pi_{f}$, there is a $\Pi u$-neighbourhood $V$ of $\alpha$ such that $\pi_{f}$ maps $V$ into $h^{-}[0, \beta)$. Also, $W=\Pi_{h \circ f}^{\leftarrow}(\beta, 1]$ is a $\Pi l$-neighbourhood of $\alpha$. By our assumption on $\alpha$, there is $x$ such that $e(x)$ is in both $V$ and $W$. This is impossible since $e(x)$ in $V$ implies $h(f(x))<\beta$ and $e(x)$ in $W$ implies $h(f(x))>\beta$.

LeMma 2. Let $\alpha$ be in the $\Pi(u \vee l)$-closure of $e[X]$ in the product $(J, u, l)^{C}$. If f and $g$ are in $C$, then so are $f \vee g, f \wedge g$ and, moreover,

$$
\begin{aligned}
& \text { (i) } \alpha_{f \vee g}=\alpha_{f} \vee \alpha_{g}, \text { (ii) } \alpha_{f \wedge g}=\alpha_{f} \wedge \alpha_{g} \text {, } \\
& \begin{array}{ll}
\alpha_{1}=1, & \text { (iv) } \alpha_{0}=0 \text {. }
\end{array}
\end{aligned}
$$

Proof. It is clear that the constant function 1 , the constant function 0 and $f \vee g$, $f \wedge g$ are all in $C$. Properties (i) to (iv) follow from the fact that $\alpha$ is in the $\Pi(u \vee l)$-closure of $e[X]$ and that $(J, u \vee l)^{C}$ is a $T_{2}$ space in which

$\pi_{f \vee g} \circ e=\pi_{f} \circ e \vee \pi_{g} \circ e, \pi_{f \wedge g} \circ e=\pi_{f} \circ e \wedge \pi_{g} \circ e, \pi_{1} \circ e=1, \pi_{0} \circ e=0$.

Proposition 1. Let $\alpha$ be in the $\Pi(u \vee l)$-closure of $e[X]$ in the product $(J, u, l)^{C}$. Let $P$ be a $\Pi u$-neighbourhood of $\alpha$. Then there is a $\{0,1\}$-valued continuous function $g:(X, T) \rightarrow(J, u)$ such that

(i) $\alpha_{g}=0$,

(ii) $g^{-}[0] \subset e^{-}[P]$.

Proof. A basic neighbourhood of $\alpha$ within $P$ is a finite intersection of sets of the form $\Pi_{f_{i}}^{-}\left[0, \gamma_{i}\right), 1 \leqslant i \leqslant n$, where $0 \leqslant \alpha_{f_{i}}<\gamma_{i}$. Fix $i$ and let $h=0$ on $\left[0, \gamma_{i}\right)$ and $h=1$ on $\left[\gamma_{i}, 1\right]$. Then $h:(J, u) \rightarrow(J, u)$. Put $g_{i}=h \circ f_{i}$. By Lemma 1 , we have $0 \leqslant \alpha_{g_{i}} \leqslant h\left(\alpha_{f_{i}}\right)=0$, so that $\alpha_{g_{i}}=0$. If we let $g=\sup \left\{g_{1}, \ldots, g_{n}\right\}$, then $g$ is in $C$ and $\alpha_{g}=\alpha_{g_{1}} \vee \cdots \vee \alpha_{g_{n}}=0$, by Lemma 2. Also, $g(x)=0$ implies $g_{i}(x)=0$ which gives $f_{i}(x)<\gamma_{i}$, so that $e(x) \in P$, as required.

COROLlary. The sets $\pi_{g}^{\leftarrow}[0]$, where $g:(X, T) \rightarrow(J, u)$ is $\{0,1\}$-valued and $\alpha_{g}=0$ form a basic system of neighbourhoods for points $\alpha$ in the $\Pi(u \vee l)$-closure of $e[X]$.

The dual result also holds.

Before proving a dual result we first state a useful remark.

REMARK. If $\alpha$ is in the $\Pi(u \vee l)$-closure of $e[X]$ in $(J, u, l)^{C}$ and $f:(X, T) \rightarrow(J, u)$ is $\{0,1\}$-valued, then $\alpha_{f}=0$ or $\alpha_{f}=1$. 
Proposition 2. Let $\alpha$ be in the $\Pi(u \vee l)$-closure of $e[X]$ in the product $(J, u, l)^{C}$ and let $Q$ be $a \prod l$-neighbourhood of $\alpha$. There is a $\{0,1\}$-valued map $g:(X, T) \rightarrow(J, u)$ such that

(i) $\alpha_{g}=1$,

(ii) $g^{\leftarrow}[1] \subset e^{\leftarrow}[Q]$.

Proof. A basic $\Pi l$-neighbourhood of $\alpha$ contained in $Q$ is a finite intersection of sets of the forms $\pi_{f_{i}}^{\leftarrow}\left(\gamma_{i}^{\prime}, 1\right], 1 \leqslant i \leqslant n$, where $f_{i}:(X, T) \rightarrow(J, u)$ and $\gamma_{i}^{\prime}<\gamma_{i}<\alpha_{f_{i}}$. Fix $i$ and let $h=0$ on $\left[0, \gamma_{i}\right)$ and $h=1$ on $\left[\gamma_{i}, 1\right]$. Then $h$ is $\{0,1\}$-valued and $h:(X, T) \rightarrow(J, u)$. Put $g_{i}=h \circ f_{i}$, so that $g_{i}:(X, T) \rightarrow(J, u)$ and is $\{0,1\}$-valued. Thus $\alpha_{g_{i}}=0$ or 1 . Suppose $\alpha_{g_{i}}=0$, then $W=\pi_{g_{i}}^{\leftarrow}[0] \cap \pi_{f_{i}}^{-}\left(\gamma_{i}, 1\right]$ is a $\Pi(u \vee l)$ neighbourhood of $\alpha$ so there is $x$ such that $e(x)$ is in $W$, hence $g_{i}(x)=0$ and $f_{i}(x)>\gamma_{i}(*)$. Now $g_{i}(x)=h\left(f_{i}(x)\right)=0$ implies $f_{i}(x)<\gamma_{i}$, which contradicts $(*)$. Thus $\alpha_{g_{i}}=1$ for $i=1,2, \ldots, n$. Let $g=\min \left\{g_{1}, \ldots, g_{n}\right\}$, then $g$ is in $C$ by Lemma 2 and $\alpha_{g}=1$. To prove (ii) let $x$ be such that $g(x)=1$. For each $i$, we have $g_{i}(x)=1$, by definition of $g$. Hence $h\left(f_{i}(x)\right)=1$, so that $\gamma_{i} \leqslant f_{i}(x) \leqslant 1$. But then $\gamma_{i}^{\prime}<f_{i}(x) \leqslant 1$, for $i=1, \ldots, n$. Hence $e(x)$ is in $Q$.

4. Open ultrafilters on $X$ and the canonical product. Let $X$ be a topological space. Following [5] the set $\theta X$ of all open ultrafilters on $X$ can be given a topology whose basic open sets are of the form $G^{*}$ which consists of all open ultrafilters which contain the open set $G$. Note that $\left(G_{1} \cup G_{2}\right)^{*}=G_{1}^{*} \cup G_{2}^{*}$ and $\left(G_{1} \cap G_{2}\right)^{*}=G_{1}^{*}$ $\cap G_{2}^{*}$. Denote this topology on $\theta X$ by $T^{*}$. $\left(\theta X, T^{*}\right)$ is a zero-dimensional compact $T_{2}$ space [5]. The absolute $\omega X$ of a space $X$ was defined by V. I. Ponomarev and is the subspace of $\theta X$ consisting of all open ultrafilters which include the neighbourhood filter of some point. The subspace of $\theta X$ consisting of all open ultrafilters which do not converge will be denoted by $\phi X$.

We now describe $\theta X, \omega X$ and $\phi X$ as subspaces of the canonical product $(J, u)^{C}$.

4.1 The characterization of $\theta X$.

Definition 1 [13]. Let $(X, P, Q)$ be a bitopological space and $A$ a subset of $X$. Let $\gamma^{*}(A)$ consist of all points $\alpha$ in $X$ such that:

(i) $\alpha$ is in the $P$-closure of $A$.

(ii) For every $Q$-neighbourhood $Q$ of $\alpha$, there is a $P$-neighbourhood $P$ of $\alpha$ such that $P \cap A \subset Q \cap A$. $A$ is $\gamma^{*}$-closed if $\gamma^{*}[A] \subset A$.

Note. (i) and (ii) imply that $\alpha$ is in the $P \vee Q$-closure of $A$.

The reader is invited to find $\gamma^{*}(A)$ for subsets $A$ of simple bitopological spaces such as $(J, u, l),(J \times J, u \times u, l \times l),(J \times J, u \times l, l \times u)$.

Proposition 3. Let $f:(X, T) \rightarrow(J, u)$ be a continuous map and $\mathcal{G}$ an open ultrafilter on $X$. Then $\{f[G] \mid G \in \mathcal{G}\}$ converges in the $u \vee$ l-topology for $J$.

Proof. Because $J$ is order complete, there are numbers $\alpha, \beta$ such that $\beta=$ $\inf \{\sup f[G] \mid G \in \mathcal{G}\}, \alpha=\sup \{\inf f[G] \mid G \in \mathcal{G}\}$. Clearly $\alpha \leqslant \beta$. We show that $\alpha<\beta$ is not possible from which it follows that $\alpha=\beta$ and, consequently, that $\{f[G] \mid G \in \mathcal{G}\}$ converges to $\alpha$ in the usual topology for $J$. Suppose $\alpha<\gamma<\beta$ for some $\gamma$ in $\mathbf{R}$. Let $G_{1}=f^{\leftarrow}[0, \gamma)$. If $G_{1}$ is in $\mathcal{G}$, then $\sup f\left[G_{1}\right] \leqslant \gamma$, contradicting our 
choice of $\beta$. If $G_{1}$ is not in $\mathcal{G}$, there is $G_{2}$ in $\mathcal{G}$ which is disjoint from $G_{1}$. But then, inf $f\left[G_{2}\right] \geqslant \gamma$, contradicting our choice of $\alpha$.

Notes. (1) Two special cases of the above proposition have been considered for bounded functions $f$-the case $J=\left(\mathbf{R}^{+}, u\right)$ in [2] and the case $\left(\mathbf{N}^{+}, u\right)$ in [4]. The above proof is different and proves both these results at once.

(2) Denote the filter $\{f[G] \mid G \in \mathcal{G}\}$ by $f^{*}[\mathcal{G}]$ and the point to which it converges (in the usual topology for $J$ ) by $s-\lim \left(f^{*}[\mathcal{G}]\right)$.

Proposition 4. Let $\mathcal{G}$ be an open ultrafilter on $X$. Let $\alpha$ be the point in $J^{c}$ given by $\alpha_{f}=s-\lim f^{*}[\mathcal{G}]$. Then $\alpha$ is in $\gamma^{*}[e[X]]$ in the product $(J, u, l)^{C}$.

Proof. (1) We first show that $\alpha$ is in the $\Pi(u \vee l)$-closure of $e[X]$ in $J^{C}$. Suppose $c<\alpha_{f}<d$. By definition of $\alpha_{f}$, there is $G$ in $\mathcal{G}$ such that $f[G] \subset(c, d)$. Because $\mathcal{G}$ is closed under finite intersections, it then follows that any $\Pi(u \vee l)$-neighbourhood of $\alpha$ intersects $e[G]$, for some $G$ in $\mathcal{G}$, as required.

(2) Let $Q$ be a $\Pi l$-neighbourhood of $\alpha$. By Proposition 2, there is a $\{0,1\}$-valued function $f:(X, T) \rightarrow(J, u)$ such that:

(i) $\pi_{f}^{-}[1]$ is a $\Pi l$-neighbourhood of $\alpha$ and

(ii) $f^{-}[1] \subset e^{-}[Q]$.

Now $\alpha_{f}=1$, so there is $G$ in $\mathcal{G}$ on which $f$ is 1 . Let $h$ be such that $h=0$ on $G, h=1$ on $X-G$. Then $h:(X, T) \rightarrow(J, u)$ is continuous and $\alpha_{h}=0$. Now $P=\pi_{h}^{-}[0]$ is a $\prod u$-neighbourhood of $\alpha$ and $e^{\leftarrow}[P]=h^{\leftarrow}[0]=f^{\leftarrow}[1] \subset e^{\leftarrow}[Q]$, so that $P \cap e[X] \subset$ $Q \cap e[X]$, as required.

Notation. Let $\sigma$ denote the map $\mathcal{G} \mapsto \alpha$ defined above. For the inverse map we have

Proposition 5. Let $\alpha \in \gamma^{*}[e[X]]$ in the product $(J, u, l)^{C}$. Let $\mathcal{G}$ consist of all sets $f^{\leftarrow}[0]$, where $f:(X, T) \rightarrow(J, u)$ is continuous, $\{0,1\}$-valued and $\alpha_{f}=0$. Then $\mathcal{G}$ is an open ultrafilter.

Proof. Because $\alpha$ is in the $\Pi u$-closure of $e[X], \mathcal{G}$ is a filter base of open sets. To see that $\mathcal{G}$ is an open ultrafilter, let $G$ be an open set in $X$ which is not in $\mathcal{G}$. We show that there is $G_{1}$ in $\mathcal{G}$ which is disjoint from $G$. Let $f=0$ on $G$ and $f=1$ on $X-G$. Then $f$ is $\{0,1\}$-valued and $f:(X, T) \rightarrow(J, u)$ so that $\alpha_{f}=1$ (because $\left.G \notin \mathcal{G}\right)$. Thus $Q=\pi_{f}^{-}[1]$ is a $\Pi l$-neighbourhood of $\alpha$. By our assumption on $\alpha$, there is a $\Pi u$-neighbourhood $P$ of $\alpha$ such that $e^{\leftarrow}[P] \subset e^{\leftarrow}[Q]$. By Proposition 1 , there is a $\{0,1\}$-valued function $h:(X, T) \rightarrow(J, u)$ such that $\alpha_{h}=0$ and $h^{\leftarrow}[0] \subset e^{\leftarrow}[P]$. Hence $h^{-}[0] \subset e^{\leftarrow}[Q]=f^{\leftarrow}[1]$, so that $h^{\leftarrow}[0]$ and $G\left(=f^{\leftarrow}[0]\right)$ are disjoint. But $h^{-}[0]$ is in $\mathcal{G}$, by definition of $\mathcal{G}$, and the proof is complete.

Notation. Let $\mu$ denote the map $\alpha \mapsto \mathcal{G}$ defined above.

Proposition 6. $\mu \circ \sigma=\mathbf{1}_{\theta x}$ and $\sigma \circ \mu=\mathbf{1}_{\gamma^{*}[e[X]]}$.

Proof. (1) Given an open ultrafilter $\mathcal{G}$, let $\alpha=\sigma(\mathcal{G})$. Consider $G \in \mathcal{G}$, let $f=0$ on $G, f=1$ on $X-G$. Then $f$ is $\{0,1\}$-valued and $f \in C$. Now $\alpha_{f}=0$, by definition of $\sigma$, so $f^{\leftarrow}[0]$ is in $\mu(\alpha)$. Hence $\mathcal{G} \subset \mu(\alpha)$. Because $\mathcal{G}$ and $\mu(\alpha)$ are ultrafilters of open sets, we have $\mathcal{G}=\mu(\alpha)$. 
(2) Given $\alpha$ in $\gamma^{*}[e[X]]$, let $\mathcal{G}=\mu(\alpha)$ and let $\beta=\sigma(\mathcal{G})$. If $\beta \neq \alpha$, then $\beta_{f} \neq \alpha_{f}$ for some $f$ in $C$. Suppose $\beta_{f}<\alpha_{f}$ then there is $h$ in $C,\{0,1\}$-valued such that $h=0$ on $\beta_{f}, h=1$ on $\alpha_{f}$. By Lemma $1, \beta_{h \circ f} \leqslant h\left(\beta_{f}\right)=0$. Because $\alpha$ and $\beta$ are in the $\Pi(u \vee v)$-closure of $e[X]$ and $h \circ f$ is $\{0,1\}$-valued, it follows that $0=\beta_{h \circ f}<\alpha_{h \circ f}$ $=1$. Let $k=h \circ f$. Now $\beta_{k}=0$ implies $k^{-}[0] \in \mathcal{G}$, otherwise $k=1$ on some member of $\mathcal{G}$ so that $s$-lim $k^{*}[\mathcal{G}]=1$; also $\pi_{k}^{-}[1]$ is a $\Pi l$-neighbourhood of $\alpha$, so there is a $\Pi u$-neighbourhood of $V$ of $\alpha$ such that $e^{\leftarrow}[V] \subset k^{\leftarrow}$ [1]. By Proposition 1, there is a $\{0,1\}$-valued $g:(X, T) \rightarrow(J, u)$ such that $\alpha_{g}=0$ and $g^{-}[0] \subset e^{-}[V]$. But then $g^{\leftarrow}[0]$ is in $\mathcal{G}$, by definition of $\mu$. This is impossible because $g^{\leftarrow}[0] \subset k^{\leftarrow}[1]$ and $k^{-}[0]$ is already in $\mathcal{G}$. An analogous argument shows that $\alpha_{f}<\beta_{f}$ is impossible. Thus $\alpha=\beta$, as required.

Finally, the continuity of $\sigma$ and $\mu$. Let $u^{*}$ denote the relativized $\Pi u$-topology on $\gamma^{*}[e[X]]$ inherited from $(J, u)^{C}$.

Proposition 7. $\sigma:\left(\theta X, T^{*}\right) \rightarrow\left(\gamma^{*}[e[X]], u^{*}\right), \mu:\left(\gamma^{*}[e[X]], u^{*}\right) \rightarrow\left(\theta X, T^{*}\right)$ are both continuous.

We omit the routine proof.

As an immediate consequence of the above we have

Proposition 8. $\left(\theta X, T^{*}\right)$ and $\left(\gamma^{*}[e[X]], u^{*}\right)$ are homeomorphic.

Corollary. $X$ is a zero dimensional compact $T_{2}$ space iff $e[X]$ is $\gamma^{*}$-closed in $(J, u, l)^{C}$.

The special case of Proposition 8 corresponding to $J=\{0,1\}$ was proved in [4] with a somewhat simpler argument corresponding to the simple structure of $\{0,1\}$ compared to $[0,1]$. It is interesting to observe that $\theta X$ is essentially $\gamma^{*}[e[X]]$ whether we consider $I^{C(X, I)}$ or $D^{C(X, D)}$.

4.2 The characterization of $\phi X$.

Definition 2. Let $(X, P, Q)$ be a bitopological space and $A \subset X$. Let $\gamma_{0}^{*}[A]$ consist of all points $\alpha$ in $\gamma^{*}[A]$ such that

(iii) For every $x$ in $A$, there is a $P$-neighbourhood of $x$ which misses $\alpha$.

A set $A$ is $\gamma_{0}^{*}$-closed if $\gamma_{0}^{*}[A] \subset A$.

Proposition 9. Let $\mathcal{G}$ be an open ultrafilter on $X . \mathcal{G}$ does not converge in $X$ if and only if $\sigma(\mathcal{G}) \in \gamma_{0}^{*}[e[X]]$ in $(J, u, l)^{C}$.

Proof. Suppose $\mathcal{G}$ does not converge to any point $X$. Given $x$, there is an open set $G_{0}$ containing $x$ which is not $\mathcal{G}$. Let $h:(X, T) \rightarrow(J, u)$ be such that $h=0$ on $G$ and $h=1$ on $X-G$. Then $\alpha_{f}=1$, since $f=1$ on some member of $\mathcal{G}$. Hence $\pi_{f}^{-}[0]$ is a $\Pi u$-neighbourhood of $e(x)$ which misses $\alpha$. Conversely, suppose $\alpha=\sigma(\mathcal{G}) \in$ $\gamma_{0}^{*}[e[X]]$. Given $x$ in $X$, there is a $\prod u$-neighbourhood of $e(x)$ which misses $\alpha$, by definition of $\gamma_{0}^{*}$. Now $(J, u, l)$ is pairwise regular, hence, so is $(J, u, l)^{C}[6]$, so that there is a $\Pi l$-neighbourhood $Q$ of $\alpha$ which misses $e(x)$. By Proposition 2 there is a $\{0,1\}$-valued function $g:(X, T) \rightarrow(J, u)$ such that $\alpha_{g}=1$ and $g^{-}[1] \subset e^{-}[Q]$. Now $\alpha_{g}=1$ implies $g=1$ on some member of $\mathcal{G}$, and $e(x) \notin Q$ shows that $g^{-}[0]$ is a neighbourhood of $x$. Hence $\mathcal{G}$ does not converge to $x$. 
The Fomin extension $\sigma(X)$ of a Hausdorff space (see [5]) is also meaningful for general topological spaces: $\sigma x=X \cup X^{\vee}$, where $X^{\vee}$ consists of all free open ultrafilters on $X$ and the neighbourhood system of a point of $\sigma X \cap X$ is its neighbourhood system in $X$, whereas a basic neighbourhood of $p \in X^{\vee}$ is $G^{*}=$ $\{p \in \sigma X \mid G \in p$, where $G$ is open in $X\}$.

We can now materialize a promise made in [10].

Proposition 10. The Fomin extension $\sigma(X)$ is homeomorphic to $e[X] \cup \gamma_{0}^{*}[e[X]]$ with the relative topology inherited from $(J, u)^{C}$.

COROllary. $X$ is almost compact if and only if $e[X]$ is $\gamma_{0}^{*}$-closed in the product $(J, u, l)^{C}$.

Note. We refer to a space as almost compact if every open ultrafilter converges (without necessarily being a Hausdorff space).

Proposition 10 and its corollary raise the question of replacing the "canonical" product $J^{C}$ by an arbitrary product of copies of $J$ in the characterization of almost compactness. The following example shows that an infinite discrete space, which is clearly not $H$-closed, can be $\gamma_{0}^{*}$-closed in a suitable product of copies of the two point space $D=\{0,1\}$.

ExAmple. Let $X$ denote the integers with the discrete topology. Clearly, $X$ is not $H$-closed. Consider the product $(D, u)^{\Lambda}$, where $\Lambda$ consists of all continuous functions $f_{n}:(X, T) \rightarrow(D, u)$ such that

$$
f_{n}(m)=0 \quad \text { if } n=m, \quad f_{n}(m)=1 \quad \text { if } n \neq m .
$$

The product map $e:(X, T) \rightarrow(D, u)^{\Lambda}$ is continuous, one-to-one and separates points and closed sets, so that $X$ and $e[X]$ are homeomorphic. Observe that the $\Pi u$-closure of $e[X]$ in the product consists of $e[X]$ and the point $\alpha$, all of whose coordinates are 1: for suppose $\beta$ is in the $\Pi u$-closure of $e[X]$ with at least one coordinate equal to zero, $\pi_{f_{n}}(\beta)=0$, say. Then $\pi_{f_{n}}^{\leftarrow}[0] \cap e[X]$ is the singleton $\{e(n)\}$, so that $\beta$ can have at most one coordinate equal to zero. Thus $\beta=e(n)$. But $\alpha$ is not in $\gamma^{*}[e[X]]$ because (ii) in the definition of $\gamma^{*}$ fails. Thus $\gamma^{*}[e[X]]$ is empty, so that $e[X]$ is $\gamma^{*}$-closed and $\gamma_{0}^{*}$-closed, as well.

4.3 The characterization of $\omega(X)$. We have shown that the free open ultrafilters on $X$ are the points $\alpha$ in $\gamma^{*}[e[X]]$ which are $\Pi l$-separated from points in $e[X]$ in the sense that for every $x$ in $X$ there is a $\Pi l$-neighbourhood of $\alpha$ which does not contain $e(x)$. Thus the convergent open ultrafilters on $X$ correspond to points which cannot be separated from all the points of $e[X]$.

Definition 3. Let $A \subset(X, P, Q)$. Let $\gamma_{00}^{*}[A]$ be the subset of $\gamma^{*}[A]$ consisting of all points $\alpha$ such that

(iii)' There is $x$ in $A$ such that every $P$-neighbourhood of $x$ contains $\alpha$.

Note. (iii)' is equivalent to the requirement that $\operatorname{cl}_{P} \alpha \cap A \neq \varnothing$, i.e. that $\alpha$ is in the $P^{*}$-closure of $A$ (in the sense of [11]).

Proposition 11. Let $\mathcal{G}$ be an open ultrafilter on $X$. $\mathcal{G}$ converges if and only if $\sigma(\mathcal{G}) \in \gamma_{00}^{*}[e[X]]$ in $(J, u, l)^{C}$. 
Proof. Suppose $\mathcal{G}$ converges to $x$. Let $\alpha \in \sigma(\mathcal{G})$. Let $P$ be an $\Pi u$-neighbourhood of $e(x)$. By Proposition 1 there is a $\{0,1\}$-valued $g:(X, T) \rightarrow(J, u)$ such that $e(x) \in$ $\pi^{\leftarrow}[0] \subset P$. But $g^{\leftarrow}[0] \in \mathcal{G}$ and $\alpha_{g}=0$ (since $g[G]=0$ for some $G$ in $\mathcal{G}$ ). Hence $\alpha \in P$.

Conversely, suppose $\alpha=\sigma(\mathcal{G})$ is in $\gamma_{00}^{*}[e[X]]$. Let $e(x) \in \operatorname{cl}_{p}(\alpha) \cap e[X]$. A basic neighbourhood of $x$ is of the form $g^{-}[0]$, where $g:(X, T) \rightarrow(J, u)$ is $\{0,1\}$-valued. For such a neighbourhood, $\pi_{g}^{-}[0]$ is a $\Pi u$-neighbourhood of $e(x)$, so that $\alpha_{g}=0$, by our assumption on $\alpha$. Thus $s-\lim g^{*}[\mathcal{G}]=0$, so that $g^{\leftarrow}[0]$ is in $\mathcal{G}$. Hence $\mathcal{G}$ converges to $x$.

COROLlaRY. $\left(\omega X, T^{*}\right)$ is homeomorphic to $\gamma_{00}^{*}[e[X]]$ with the relative topology inherited from the product $(J, u)^{C}$.

5. Almost compact spaces and maximal separation properties. In [2] Frolık and Liu characterized $H$-closed $T_{2}$ spaces as maximal separated spaces in their closure in the canonical product $(I, u)^{C((X, T),(I, u))}$. An analogous characterization was obtained by K. Halpin [4] where $(I, u)$ is replaced by the simpler $(D, u)$. In this section we present a unified proof of both of these results together with a generalization to spaces which are not necessarily separated.

Definition 4. Let $X$ be a toplogical space and $A \subset X . A$ is said to be relatively separated with respect to $X$ if for every $a \in A$ and $x \in X-A$ there is a neighbourhood of $a$ and a neighbourhood of $x$ which do not intersect.

Note. This notation of relative separation is closely related to Liu's notion of " $T_{2}$ except for $A$ " [7] but it does not require that $X-A$ be a $T_{2}$ space.

Proposition 12. Let $X$ be a topological space. The following are equivalent:

(1) $X$ is almost compact.

(2) $e[X]$ is maximal relatively separated with respect to the $\Pi u$-closure of $e[X]$ in $(J, u)^{C}$.

(3) $e[X]$ is maximal relatively separated with respect to the $\Pi(u \vee l)$-closure of $e[X]$ in $(J, u)^{C}$.

(4) $e[X]$ is maximal relatively separated in $e[X] \cup \gamma^{*}[e[X]] \subset(J, u)^{C}$.

Proof. (1) $\Rightarrow(2)$. Suppose $\alpha \in \operatorname{cl}[e[X]]-e[X]$ and that $e[X]$ is relatively separated in $e[X] \cup\{\alpha\}$. The sets $e^{\leftarrow}[V]$, where $V$ is a $\prod u$-open neighbourhood of $\alpha$, form an open filter base $F$ in $X$. Let $x$ be an adherence point of $F$. By our assumption, there is a $\Pi u$-neighbourhood $W$ of $e[x]$ which is disjoint from the neighbourhood $V$ of $\alpha$. But then $e^{\leftarrow}[W]$ is a neighbourhood of $x$ which is disjoint from $e^{-}[V]$ in $F$, which is impossible.

$(2) \Rightarrow(3)$ and $(3) \Rightarrow(4)$ are clear.

(4) $\Rightarrow$ (1). Suppose $X$ is not almost compact. Then there is an open filter $\mathcal{G}$ on $X$ which does not converge. Let $\alpha=\sigma(\mathcal{G})$, then $\alpha \in \gamma^{*}[e[X]]$ by Proposition 4 . We now show that $e[X]$ is relatively separated in $e[X] \cup\{\alpha\}$ which contradicts (4) and completes the proof. Given $x \in X$, there are disjoint open sets $G_{0}$ and $G_{1}$ such that 
$x \in G_{0}, G_{1} \in \mathcal{G}$. Let $f_{i}:(X, T) \rightarrow(J, u)$ be such that $f_{0}=0$ on $G_{0}, f_{0}=1$ off $G_{0}$; $f_{1}=0$ on $G_{1}, f_{1}=1$ off $G_{1}$. Now $\pi_{f_{0}}^{\leftarrow}[0] \cap(e[X] \cup\{\alpha\})$ is a neighbourhood of $e(x)$ and $\pi_{f_{1}}^{-}[0] \cap(e[X] \cup\{\alpha\})$ is a disjoint neighbourhood of $\alpha$, as required.

When $X$ is $T_{2}$, it is possible to sharpen the characterization of $H$-closed spaces given by Frolík and Liu [2] $(J=[0,1])$ and by K. Halpin [4] $(J=\{0,1\})$. Our characterization is a straightforward consequence of Proposition 12.

Proposition 13. Let $X$ be a $T_{2}$ space. The following are equivalent:

(1) $X$ is $H$-closed.

(2) $e[X]$ is maximal $T_{2}$ in its closure in $(J, u)^{C}$.

(3) $e[X]$ is maximal $T_{2}$ in its $\Pi(u \vee l)$-closure in $(J, u)^{C}$.

(4) $e[X]$ is maximal $T_{2}$ in $e[X] \cup \gamma^{*}[e[X]]$ in $(J, u)^{C}$.

6. $H$-closed extensions in the canonical product. Suppose $X$ is not almost compact. We have seen that $e[X]$ is not a maximal relatively separated subspace of its closure in the canonical product. However, Zorn's lemma shows that there is a subspace of the closure of $e[X]$, which contains $e[X]$ and is maximally separated in its closure in the product. Let us call such a space $\left(m(X), u^{*}\right)$, where $u^{*}$ denotes the restriction of the product topology on $(J, u)^{C}$ to $m(X)$.

Proposition 14. $\left(m(X), u^{*}\right)$ is almost compact.

Proof. Let $\mathcal{G}_{1}$ be an open ultrafilter on $m(X) \cdot \mathcal{G}_{1}$ determines an open ultrafilter $\mathcal{G}_{0}$ on $e[X]$ consisting of all open sets $G \cap e[X], G \in \mathcal{G}_{1}$. Then $\mathcal{G}=e^{\leftarrow}\left[\mathcal{G}_{0}\right]$ is an open ultrafilter on $X$. Let $\alpha=\sigma(\mathcal{G})$ be the point in the canonical product determined by $\mathcal{G}$. We now show that $\mathcal{G}_{1}$ converges to $\alpha$, which is in the $\Pi(u \vee l)$-closure of $e[X]$. Let $V$ be a basic $\Pi u$-neighbourhood of $\alpha$. By Proposition 1 , there is a $\{0,1\}$-valued $f:(X, T) \rightarrow(J, u)$ such that $V=\pi_{f}^{\leftarrow}[0]$. By definition of $\alpha$, it follows that $e^{-}\left[\pi_{f}^{-}[0]\right]=f^{-}[0] \in \mathcal{G}$. Hence $\pi_{f}^{\leftarrow}[0] \cap e[X] \in \mathcal{G}_{0}$, so that $\pi_{f}^{\leftarrow}[0] \in \mathcal{G}_{1}$. Thus $\mathcal{G}_{1}$ converges to $\alpha$. Finally, $\alpha$ must be in $m(X)$, otherwise $e[X]$ is a relatively separated subspace of $m(X) \cup\{\alpha\}$ (as is easily checked), contradicting the maximality of $m(X)$.

It is interesting that the Fomin extension of a $T_{2}$ space is amongst one of these maximal $T_{2}$ subspaces of the product $(J, u)^{C}$.

Proposition 15. The Fomin extension $\sigma X$ of a $T_{2}$ space $X$ is a maximal $T_{2}$ subspace of the closure of $e[X]$ in the product $(J, u)^{C}$.

Proof. By Proposition 10, $\sigma(X)$ is homeomorphic to $e[X] \cup \gamma_{0}^{*}[e[X]]=m(X)$. Suppose $m(X)$ is not a maximal $T_{2}$ subspace of the $\prod u$-closure of $e[X]$ in $(J, u)^{C}$. Then there is $\alpha$ in the $\prod u$-closure of $e[X]$ such that $m(X) \cup\{\alpha\}(\neq m(X))$ is a $T_{2}$ subspace of $(J, u)^{C}$. Now $\alpha$ determines an open filter $\mathcal{G}_{0}$ consisting of all sets $e^{-}[V]$ where $V$ is a $\Pi u$-neighbourhood of $\alpha$. Let $\mathcal{G}$ be an open ultrafilter which contains $\mathcal{G}_{0}$. Because $e[X] \cup\{\alpha\}$ is $T_{2}$, it follows that $\mathcal{G}$ does not converge in $X$. Hence $\beta=\sigma(\mathcal{G})$ is in $\gamma_{0}^{*}[e[X]]$. But every $\Pi u$-neighbourhood of $\alpha$ contains $\beta$ : For suppose $\pi_{f}^{\leftarrow}[0, c)$ is a subbasic neighbourhood of $\alpha$ which does not contain $\beta$. Then $\Pi_{f}(\beta)=$ $s-\lim \left(f^{*}[\mathcal{G}]\right) \geqslant c$. Let $d$ be such that $\pi_{f}(\alpha)<d<c$, then there is $G$ in $\mathcal{G}$ such that 
$f[G] \subset(d, \infty)$. But $e^{\leftarrow}\left[\pi_{f}^{\leftarrow}[0, d)\right]$ is in $\mathcal{G}_{0}$ and is disjoint from $G$, which is impossible. Thus $m(X) \cup\{\alpha\}$ is not even $T_{1}$, contrary to our assumption.

7. Almost realcompactness. Let $\kappa$ be an infinite cardinal. A filter $\mathcal{G}$ is said to have the $\kappa$-intersection property if every subcollection of fewer than $\kappa$ members of $\mathcal{G}$ has nonempty intersection. A space $X$ is $\kappa$-almost compact if whenever $\mathcal{G}$ is an open ultrafilter such that the family of closures of its members has the $\kappa$-intersection property then $\mathcal{G}$ converges. It is possible to extend the previous results to characterize $\kappa$-almost compactness. However we have not been able to find, for $\kappa>\boldsymbol{\kappa}_{1}$, a space $J_{\kappa}$ such that $X$ is $\kappa$-almost compact if and only if $e[X]$ is maximal $T_{2}$ in its closure in the product $\left(J_{\kappa}, u\right)^{C\left(X, J_{\kappa}\right)}$ when $X$ is $T_{2}$.

For $\kappa=\boldsymbol{\aleph}_{1}$, Frolík [1] has called the resulting $\kappa$-almost compact space, almost realcompact. These spaces have been characterized as follows: a $T_{2}$ space $X$ is almost realcompact if and only if $e[X]$ is maximal $T_{2}$ in its closure in the canonical product $(J, u)^{C}$. The case $J=\mathbf{R}^{+}$was proved by Frolik and Liu [2] and the case $J=\mathbf{N}^{+}$by K. Halpin [4]. In this section, we shall again give a unified proof of both these characterizations and extend these results by dropping the requirement that $X$ be a $T_{2}$ space.

In what follows let $J_{1}=\mathbf{N}^{+}$or $\mathbf{R}^{+}$, and let $C_{1}$ denote the set of continuous functions $f:(X, T) \rightarrow\left(J_{1}, u\right)$.

Proposition 3'. Let $f:(X, T) \rightarrow\left(J_{1}, u\right)$ be a continuous map and $\mathcal{G}$ an open ultrafilter with the countable closed intersection property. Then $f$ is bounded on some member of $\mathcal{G}$ and $\{f[G] \mid G \in \mathcal{G}\}$ converges in the $u \vee$ l-topology for $J_{1}$.

Proof. We first show that $f$ must be bounded on some member of $\mathcal{G}$. The result then follows from Proposition 3. Suppose $f$ is not bounded on any member of $\mathcal{G}$. Then, for each $n=1,2,3, \ldots$, there is $G_{n} \in \mathcal{G}$ such that $f^{\leftarrow}[0, n) \cap G_{n}=\varnothing$. Let $x \in \cap\left\{\bar{G}_{n} \mid n=1,2, \ldots\right\}$. Let $N$ be an integer larger than $f(x)$. Then $f^{\leftarrow}[0, N) \cap$ $G_{N} \neq \varnothing\left(\right.$ since $\left.x \in \bar{G}_{N}\right)$, which is a contradiction.

Note. Proposition $3^{\prime}$ is Theorem 1 and Lemma 2 of [3]. We give our proof because it is more direct.

Proposition 5'. Let $\alpha \in \gamma^{*}[e[X]]$ in $\left(J_{1}, u, l\right)^{C_{1}}$. Let $\mathcal{G}$ consist of all sets $f^{-}[0]$, where $f:(X, T) \rightarrow(J, u)$ is continuous, $\{0,1\}$-valued and $\alpha_{f}=0$. Then $\mathcal{G}$ is an open ultrafilter with the countable closed intersection property.

Proof. Proposition 5 establishes that $\mathcal{G}$ is an open ultrafilter. To show that it has the countable closed intersection property, suppose, on the contrary, that there are sets $G_{n}$ in $\mathcal{G}$ such that $G_{1} \supset G_{2} \supset \cdots \supset G_{n} \supset \cdots$ and $\cap\left\{\bar{G}_{n} \mid n=1,2, \ldots\right\}=\varnothing$. Define $f: X \rightarrow J_{1}$ by $f(x)=\inf \left\{n \mid x \notin \bar{G}_{n}\right\}$. Clearly, $f:(X, T) \rightarrow\left(J_{1}, u\right)$ is continuous. Suppose $\alpha_{f}=m$, then $\alpha_{(f \vee m)-m}=0$ (by an extension of Lemma 2). By the definition of $\mathcal{G}$, we have $((f \vee m)-m)^{\leftarrow}[0]=f^{\leftarrow}[0, m]$ is in $\mathcal{G}$. Now, $\mathcal{G}$ is a filter, so there is $x \in f^{\leftarrow}[0, m] \cap G_{m}$, which is impossible because $x \in G_{m}$ implies $f(x)>m$.

Notation. Let $\sigma^{\prime}$ denote the map from $\gamma^{*}[e[X]]$ onto the open ultrafilters on $X$ with the countable closed intersection property. The space of open ultrafilters with the countable closed intersection property regarded as a subspace of $\theta(X)$ will be denoted by $\theta^{\prime}(X)$. 
Proposition $8^{\prime} .\left(\theta^{\prime}(X), T^{*}\right)$ and $\left(\gamma^{*}[e[X]], u^{*}\right)$ are homeomorphic.

Proposition 9'. Let $\mathcal{G}$ be an open ultrafilter on $X$ with the countable closed intersection property. $\mathcal{G}$ does not converge if and only if $\sigma^{\prime}(\mathcal{G}) \in \gamma_{0}^{*}[e[X]]$ in $\left(J_{1}, u, l\right)^{C_{1}}$.

There is an almost realcompact analogue of the Fomin extension, $\sigma^{\prime} X$, which is a subspace of the Fomin extension $\sigma X=X \cup X^{\vee}$ consisting of $X$ and all nonconvergent open ultrafilters on $X$ with the countable closed intersection property. It is easy to prove directly that $\sigma^{\prime} X$ is in fact almost realcompact.

In [8], Liu and Strecker studied another almost realcompact extension of a Hausdorff space which is related to ours as the Katětov extension is to the Fomin extension.

Proposition $10^{\prime}$. The almost realcompact extension $\sigma^{\prime} X$ is homeomorphic to e[X] $\cup \gamma_{0}^{*}[e[X]]$ with the relative topology induced by $\left(J_{1}, u\right)^{C_{1}}$.

COROllaRY. $X$ is almost realcompact if and only if $e[X]$ is $\gamma_{0}^{*}$-closed in the product $\left(J_{1}, u, l\right)^{C}$.

Proposition 12'. Let $X$ be a topological space. The following are equivalent:

(1) $X$ is almost realcompact.

(2) $e[X]$ is maximal relatively separated in its $\prod u$-closure in the product $\left(J_{1}, u\right)^{C_{1}}$.

(3) $e[X]$ is maximal relatively separated in its $\Pi(u \vee l)$-closure in the product $\left(J_{1}, u\right)^{C_{1}}$.

(4) $e[X]$ is maximal relatively separated in $e[X] \cup \gamma^{*}[e[X]]$ in the product $\left(J_{1}, u\right)^{C_{1}}$.

Proof. In view of Proposition 12, the only implication that requires justification is $(1) \Rightarrow(2)$. Assume $X$ is almost realcompact and suppose there is $\alpha$ in $\Pi u$-closure $[e[X]]-e[X]$ such that $e[X]$ relatively separated in $e[X] \cup\{\alpha\}$. As in Proposition 12 , let $\mathscr{F}$ be the open filter of sets $e^{-}[V]$ where $V$ is a $\Pi u$-neighbourhood of $\alpha$. Let $\mathcal{G}$ be an open ultrafilter containing $\mathscr{F}$. We establish the (remarkable) fact that $\mathcal{G}$ has the countable closed intersection property, and the proof then proceeds as in Proposition $12,(1) \Rightarrow(2)$. Suppose $\mathcal{G}$ does not have the countable closed intersection property. Let $G_{1} \supset G_{2} \supset \cdots \supset G_{n} \supset \cdots$ be sets in $\mathcal{G}$ whose closures have an empty intersection. As in Proposition 5', there is $f:(X, T) \rightarrow\left(J_{1}, u\right)$ given by $f(x)=$ $\inf \left\{n \mid x \notin \bar{G}_{n}\right\}$. Suppose $\alpha_{f}=m$. Then $V=\pi^{-}[0, m+1)$ is a neighbourhood of $\alpha$, so that $f^{-}[0, m+1)=e^{\leftarrow}[V]$ is in $\mathscr{F}$. This is impossible, since $f^{-}[0, m+1) \cap G_{m}=\varnothing$.

Proposition 13'. Let $X$ be a $T_{2}$ space. The following are equivalent:

(1) $X$ is almost realcompact.

(2) $e[X]$ is maximal $T_{2}$ in its closure in $\left(J_{1}, u\right)^{C_{1}}$.

(3) $e[X]$ is maximal $T_{2}$ in its $\Pi(u \vee l)$-closure in $\left(J_{1}, u\right)^{C_{1}}$.

(4) $e[X]$ is maximal $T_{2}$ in $e[X] \cup \gamma^{*}[e[X]]$ in $\left(J_{1}, u\right)^{C_{1}}$. 


\section{REFERENCES}

1. Z. Frolik, A generalization of realcompact spaces, Czechoslovak Math. J. 13 (88) (1963), 127-138. MR 27\#5224

2. Z. Frolik and Chen Tung Liu, An embedding characterization of almost real compact spaces, Proc. Amer. Math. Soc. 32 (1972), 294-298. MR 44 \# 7518

3. L. Gillman and M. Jerison, Rings of continuous functions, Van Nostrand, Princeton, N.J., 1960. MR 22 \#6994

4. K. Halpin, H-closed spaces and almost compact spaces, M. Sc. thesis, University of Cape Town, 1974.

5. S. Iliadis and S. Fomin, The methods of centered systems in the theory of topological spaces, Russian Math. Surveys 21 (1966), 37-62. MR 34 \#3526

6. J. C. Kelly, Bitopological spaces, Proc. London Math. Soc. 13 (1963), 71-89. MR 26 \#29

7. Chen Tung Liu, Absolutely closed spaces, Trans. Amer. Math. Soc. 130 (1968). MR 36 \#2107

8. C. T. Liu and G. E. Strecker, Concerning almost realcompactifications, Czechoslovak Math. J. 22 (97) (1972), 181-190. MR 46 \#2644

9. G. M. Murdeshwar and S. A. Naimpally, Quasi-uniform topological spaces, Noordhoff, Amsterdam, 1966. MR 35 \#2267

10. J. R. Porter, Categorical problems in minimal spaces, Proc. Conf. Categorical Topology, Mannheim 1975, Lecture Notes in Math., vol. 540, Springer-Verlag, Berlin, 1976. MR 53 \# 11558

11. S. Salbany, Bitopological spaces, compactifications and completions, Math. Monographs, University of Cape Town, vol. 1. MR 54 \# 13869

12. __ Reflective subcategories and closure operators, Proc. Conf. Categorical Topology, Mannheim, 1975, Lecture Notes in Math., vol. 540, Springer-Verlag, Berlin, 1978. MR 53 \#11558

13. An embedding characterization of compact spaces, (Proc. Conf. Categorical Topology, Berlin, 1978), Lecture Notes in Math., vol. 719, Springer-Verlag, Berlin, 1979.

Department of Mathematics, University of ZimbabWe, Harare, Zimbabwe 